Pacific Journal of Mathematics

BOUNDED ANALYTIC FUNCTIONS ON A CLASS OF OPEN 


\title{
BOUNDED ANALYTIC FUNCTIONS ON A CLASS OF OPEN RIEMANN SURFACES
}

\author{
Charles M. Stanton
}

\begin{abstract}
In this paper some function theoretic properties of an open Riemann surface are related to a condition on the exhaustion of the surface by finite Riemann surfaces. The class of Myrberg surfaces is introduced; these are certain branched covering surfaces of the unit disc. The exhaustion condition is used to distinguish those Myrberg surfaces on which the bounded analytic functions separate points. A complete description is given of the ways in which the space of bounded analytic functions on a Myrberg surface can degenerate. The exhaustion condition is stated in terms of the Green's function; it is already known to be equivalent to a function theoretic condition on the fundamental group of the surface. This latter condition is shown to imply that the surface is an open subset of the spectrum of its Banach algebra of bounded analytic functions.
\end{abstract}

Widom [14], [15] introduced the exhaustion condition for open Riemann surfaces and discussed its relation to the fundamental group. Plane domains with similar properties had been considered by Voichick [13]. In $\S 1$, the relevant parts of Widom's work are summarized. In $\S 2$, we consider a class of finitely sheeted branched coverings of the unit disc, following a construction of Myrberg [10]. Many examples of function theoretic difficulties on surfaces of infinite genus are based on this construction. (Cf. Hejhal [9] and Sario and Nakai [11].) These examples exploit the failure of the bounded analytic functions to separate points on the surface. We show that Widom's condition distinguishes those Myrberg surfaces on which the bounded analytic functions separate points from those on which all bounded analytic functions are lifted from another Myrberg surface. In $\S 3$ we use the relationship of Widom's condition to the fundamental group to prove that Widom's condition implies the surface is an open subset of the spectrum of its Banach algebra of bounded analytic functions. This proof follows an argument of Stout [9] in making use of a fact about analytic structure in spectra.

The author would like to thank the members of the Brown Analysis Seminar, in particular Professors Accola, Cole and Wermer, for many helpful discussions of this material. The author is grateful to Professor T. W. Gamelin for enlightening correspondence on the contents of this paper. The author is also indebted to the referee for his criticism of previous versions of the paper. 
1. Widom's condition. The material in this section is due to Widom [14], [15] and a more complete development can be found in those papers. Let $W$ be an open Riemann surface, $H^{\infty}(W)$ its Banach algebra of bounded analytic functions, $\pi_{1}(W)$ its fundamental group, and $\pi_{1}(W)^{*}$ the character group of $\pi_{1}(W)$. That is, $\pi_{1}(W)^{*}$ is the group of all homomorphisms of $\pi_{1}(W)$ into the circle group. Let $H_{1}(W)$ be the first singular homology group of $W$ with integer coefficients, and let $H_{1}(W)^{*}$ be the character group of $H_{1}(W)$. Since $H_{1}(W)$ is $\pi_{1}(W)$ made abelian, $\pi_{1}(W)^{*}$ is naturally isomorphic to $H_{1}(W)^{*}$.

Each character of $\pi_{1}(W)$ arises by analytic continuation of a multiple-valued analytic function with single-valued modulus. For let $F$ be a multiple-valued analytic function on $W$ such that $|F|$ is single-valued. If $C$ is a closed curve on $W$, analytic continuation of a function element of $F$ around $C$ results in multiplying the function element by a constant $\alpha$ of modulus one. Now $\alpha$ depends only on $F$ and the homotopy class of $C$. Thus the equation $\chi_{F}(C)=\alpha$ defines a mapping of the fundamental group into the circle, and this mapping is a character. Conversely, each $\chi$ in $\pi_{1}(W)^{*}$ is naturally identified with an element of $H_{1}(W)^{*}$, which we also denote by $\chi$. The BehnkeStein theorem guarantees the existence of a real-valued harmonic function $U$ on $W$ whose conjugate differential satisfies

$$
\exp \left\{i \int_{C} * d u\right\}=\chi(C)
$$

for every cycle $C$ on $W$. Let $\tilde{u}$ denote the multiple-valued harmonic conjugate of $u$ and set $F=\exp \{u+i \widetilde{u}\}$. Then $F$ is a multiplevalued analytic function on $W,|F|$ is single-valued, and $\chi_{F}=\chi$.

Widom gives a necessary and sufficient condition that every character of $\pi_{1}(W)$ come from a multiple-valued analytic function with bounded single-valued modulus. For $\chi$ in $\pi_{1}(W)^{*}$, let $H(W, \chi)$ be the class of all multiple-valued analytic functions $F$ on $W$ such that $|F|$ is single-valued and $\chi_{F}=\chi$. For $q$ in $W$, set

$$
\begin{aligned}
m(W, q, \chi) & =\sup \{|F(q)|: F \in H(W, \chi),|F| \leqq 1 \text { on } W\} \\
m(W, q) & =\inf \left\{m(W, q, \chi): \chi \in \pi_{1}(W)^{*}\right\}
\end{aligned}
$$

with the convention that zero is the supremum of an empty set of nonnegative real numbers.

Assume that $W$ is hyperbolic and let $g(p, q)$ be the Green's function of $W$ with pole at $q$. For each $\alpha>0$, let $W_{\alpha}=W_{\alpha}(q)=\{p \in W: g(p$, $q)>\alpha\}$. Now $W_{\alpha}$ is an open connected subset of $W$. For assume that $W_{\alpha}$ is the union of two disjoint open subregions $V_{1}$ and $V_{2}$, where $q$ is in $V_{1}$. Define $h$ on $W$ by setting $h(p)=g(p, q)$ for $p$ in $W \sim V_{2}$ and $h(p)=\alpha$ for $p$ in $V_{2}$. Then $h$ is a nonnegative super- 
harmonic function on $W$ with a positive logarithmic singularity at $q$. Since $h(p) \leqq g(p, q)$, it follows from the minimum property of the Green's function that $h(p)=g(p, q)$. Hence $V_{2}$ is empty.

Let $\beta(\alpha)$ be the first Betti number of $W_{\alpha}$. Widom establishes the formula

$$
m(W, q)=\exp \left\{-\int_{0}^{\infty} \beta(\alpha) d \alpha\right\}
$$

with the convention that the right hand side is zero when the integral diverges. Widom also proves that convergence of the integral does not depend on the choice of the point $q$, and is a property of the ideal boundary of $W$. Widom proves that every character of $\pi_{1}(W)$ arises from a multiple-valued analytic function with bounded singlevalued modulus if and only if $m(W)>0$. Moreover, if $m(W)>0$, $q$ is in $W$, and $\chi$ is in $\pi_{1}(W)^{*}$ then there is an $F$ in $H(W, \chi)$ such that $|F|$ is bounded and $F(q) \neq 0$.

Assume that $m(W)>0$ and let $\widetilde{g}(p, q)$ be the multiple-valued harmonic conjugate of $g(p, q)$. Form the function

$$
\Phi(p, q)=\exp -\{g(p, q)+i \widetilde{g}(p, q)\} .
$$

Fixing $q$ and considering $\Phi(p, q)$ as a function of $p$, we find that it has the following properties: $\Phi$ is a multiple-valued analytic function of $p$ with single-valued modulus; $|\Phi|<1$ on $W ; \Phi$ vanishes only at $q$, where it has a simple zero. Let $\chi$ be the character of $\pi_{1}(W)$ corresponding to $\Phi$, and let $\chi^{-1}$ be the inverse character. For each $p \neq q$ in $W$, there is a bounded function $\Psi$ in $H\left(W, \chi^{-1}\right)$ such that $\Psi(p) \neq 0$. Let $f=\Phi \Psi$. Then $f$ is in $H^{\infty}(W), f(p) \neq 0$ and $f(q)=$ 0 . Hence $H^{\infty}(W)$ separates the points of $W$. (This argument is due to Widom [14], [15].)

2. Myrberg surfaces and Widom's condition. We shall call a Riemann surface $W$ a Myrberg surface over the unit disc $U$ (or more simply a Myrberg surface) if there is an analytic function $z: W \rightarrow U$ realizing $W$ as an $n$-sheeted, branched, full covering surface of $U$. (That is, each point of $U$ has exactly $n$ pre-images, counting multiplicities.)

Our first goal is the following theorem.

TheOREM 1. Let $W$ be a Myrberg surface over the unit disc. $A$ necessary and sufficient condition that $H^{\circ}(W)$ separate the points of $W$ is that $m(W)>0$. This holds precisely when the projections of the branch points form the zero set of a Blaschke product. 
Proof. We have seen that $H^{\infty}$ separates the points of $W$ if $m(W)>0$. The converse follows immediately from the following two lemmas.

Lemma 2. Let $(W, z)$ be a Myrberg surface over $U$. Let $\left\langle a_{k}\right\rangle$ be the sequence of points of $U$ over which $z$ is branched. For each $k$, let $n_{k}$ be the order of branching over $a_{k}$. If $H^{\infty}(W)$ separates the points of $W$, then the series $\Sigma n_{k} \log \left|a_{k}\right|$ converges.

LEMma 3. In the notation of Lemma 2, a necessary and sufficient condition that $m(W)>0$ is that the series $\Sigma n_{k} \log \left|a_{k}\right|$ converge.

Proof of Lemma 2. Let $a$ be a point of $U$ such that $z^{-1}[a]$ consists of $n$ distinct points of $W$. Since $H^{\infty}(W)$ separates the points of $W$, there exists an $f$ in $H^{\infty}(W)$ which takes $n$ distinct values at the points of $z^{-1}[a]$. By a standard argument borrowed from the theory of compact Riemann surfaces, $f$ satisfies an equation of the form

$$
f^{n}+a_{1}(z) f^{n-1}+\cdots+a_{n}(z)=0
$$

where $a_{1}, \cdots, a_{n}$ are in $H^{\infty}(U)$. Let $D$ be the discriminant of this equation. Then $D$ is in $H^{\infty}(U), D$ does not vanish identically, and $D$ vanishes at each point $a_{k}$ to order at least $n_{k}$. Hence the series $\Sigma n_{k} \log \left|a_{k}\right|$ converges.

Proof of Lemma 3. To determine the convergence of Widom's integral for $W$, we may suppose that there are $n$ distinct points $q_{k}, 1 \leqq k \leqq n$, of $W$ with $z\left(q_{k}\right)=0$. Set

$$
h(p)=n^{-1} \sum_{k=1}^{n} g\left(p, q_{k}\right), \quad p \in W .
$$

Then $h(p)=-\log |z(p)|$. We shall show that Widom's integral converges if and only if the corresponding integral formed from $h$ converges.

It is sufficient to discuss Widom's integral for the point $q_{1}$. We use our previously established notation $W_{\alpha}$ and $\beta(\alpha)$ with respect to this point. For each $\alpha>0$ we let $V_{\alpha}=\{p \in W: h(p)>\alpha\}$ and $\gamma(\alpha)$ the first Betti number of $V_{\alpha}$. Fix $R, 0<R<1$, such that $\{p \in W$ : $|h(p)|>R\}$ is connected. There is a constant $A$ such that

$$
A^{-1} g\left(p, q_{1}\right) \leqq h(p) \leqq A g\left(p, q_{1}\right), \quad|z(p)| \geqq R .
$$

If $0<\alpha<-\log R$, then $V_{\alpha}$ is connected and we have that $W_{\alpha A} \subset$ 
$V_{\alpha} \subset W_{\alpha A^{-1}}$. It follows from the maximum principle that $V_{\alpha} \sim W_{\alpha A}$ has no components which are relatively compact in $V_{\alpha}$. Thus every bounding cycle in $W_{\alpha A}$ already bounds in $V_{\alpha}$, so $\beta(\alpha A) \leqq \gamma(\alpha)$. Similarly $\gamma(\alpha)<\beta\left(\alpha A^{-1}\right)$. Thus, for every $\varepsilon>0$,

$$
A^{-1} \int_{A \varepsilon}^{-A \log R} \beta(\alpha) d \alpha \leqq \int_{\varepsilon}^{-\log R} \gamma(\alpha) d \alpha \leqq A \int_{A^{-1_{\varepsilon}}}^{-A^{-1} \log R} \beta(\alpha) d \alpha
$$

Therefore $\int_{0}^{\infty} \beta(\alpha) d \alpha$ converges if and only if $\int_{0}^{\infty} \gamma(\alpha) d \alpha$ converges.

We next relate the convergence of the second integral to the branching of the mapping $z$. Now

$$
V_{\alpha}=\left\{p \in W:|z(p)|<e^{-\alpha}\right\} .
$$

If $0<\alpha<-\log R$ and no branch point of $z$ lies over the circle $|z|=e^{-\alpha}$, we can form the double $\hat{V}_{\alpha}$ of $V_{\alpha}$. Now $z$ extends to be a meromorphic function of valence $n$ on $\hat{V}_{\alpha}$, and $\gamma(\alpha)$ is the genus of $\hat{V}_{\alpha}$. By the Riemann-Hurwitz formula

$$
\gamma(\alpha)=b\left(e^{-\alpha}\right)+1-n
$$

where $b(r)$ is the number of branch points over the disc $|z|<r$. This formula still holds even if there are branch points over $|z|=$ $e^{-\alpha}$. Now we have

$$
\int_{0}^{\infty} \gamma(\alpha) d \alpha=\left.\alpha \gamma(\alpha)\right|_{0} ^{\infty}-\int_{0}^{\infty} \alpha d \gamma(\alpha)
$$

Changing variables, we see that

$$
\int_{0}^{\log a} \alpha d \gamma(\alpha)=-\int_{a}^{1} \log r d b(r)=\sum_{a<\left|a_{k}\right|<1} n_{k} \log \left|a_{k}\right|,
$$

for $b(r)$ is a saltus function whose jump at $r$ is the total order of branching over $|z|=r$. This series differs from the series in the lemma by finitely many terms. Thus, letting $a$ tend to zero, we have the lemma.

For later use, we extract the following somewhat technical corollary from the proof of Theorem 1.

CoRollary. Let $(W, z)$ be a Myrberg surface over the unit disc $U$. If for some point a in $U$ there exists a function $f$ in $H^{\infty}(W)$ such that $z$ is unbranched over $a$ and $f$ separates the points of $z^{-1}[a]$, then $m(W)>0$.

Theorem 1 raises the question of how the bounded analytic functions on a Myrberg surface can degenerate. We prove the following theorem. 
THeOREM 2. Let $W$ be a Myrberg surface over the unit disc. Then there exist a Myrberg surface $W_{1}$ over the unit disc and an analytic mapping $\Phi$ of $W$ onto $W_{1}$ having the following properties:

(i) $H^{\infty}\left(W_{1}\right)$ separates the points of $W_{1}$;

(ii) for each $f$ in $H^{\infty}(W)$ there is an $f_{1}$ in $H^{\infty}\left(W_{1}\right)$ such that $f=f_{1} \cdot \Phi$.

Proof. Now $z$ maps $W$ onto $U$. For each $\zeta$ in $U$ and each $f$

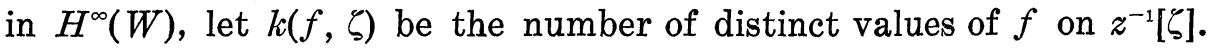
Let $k$ be the largest of these integers. Fix $\zeta_{0}$ in $U$ and $g$ in $H^{\infty}(W)$ such that $k\left[g, \zeta_{0}\right]=k$. Now $k[g, \zeta]=k$ for all $\zeta$ in some neighborhood of $\zeta_{0}$, so we may also assume that $z$ is unbranched over $\zeta_{0}$. There are $k$ function elements associated with $g$ and lying over $\zeta_{0}$. For any other point $\zeta$ of $U$ there are at most $k$ of these function elements over $\zeta$. If, in addition, $z$ is unbranched over $\zeta$ then there are exactly $k$ function elements over $\zeta$. For $\zeta_{0}$ may be joined to $\zeta$ by a curve over no point of which is $z$ branched. Each of the $k$ function elements at $\zeta_{0}$ can then be continued along this curve, producing $k$ function elements at $\zeta$. Let $W_{1}$ be the Riemann surface formed from these function elements. Then $W_{1}$ is a $k$-sheeted covering of $U$ with projection $z_{1}: W_{1} \rightarrow U$. Hence $\left(W_{1}, z_{1}\right)$ is a Myrberg surface over $U$.

Define a mapping $\Phi$ from $W$ to $W_{1}$ as follows: Each point of $W$ yields a function element of $g$; we map that point of $W$ to the corresponding function element. The mapping $\Phi$ so defined is an analytic mapping of $W$ onto $W_{1}$, and $z_{1} \circ \Phi=z$. There is also a function $g_{1}$ in $H^{\circ}\left(W_{1}\right)$ such that $g=g_{1} \circ \Phi$. Since $g_{1}$ separates the points of $W_{1}$ which lie over $\zeta_{0}$, it follows from the Corollary to Theorem 1 that $m\left(W_{1}\right)>0$. Thus $H^{\circ}\left(W_{1}\right)$ separates the points of $W_{1}$.

Let $f$ be in $H^{\circ}(W)$. Again borrowing an argument from the theory of compact Riemann surfaces, there exist meromorphic functions $b_{1}, \cdots, b_{k}$ in $U$ such that

$$
f(p)=b_{1}(z(p)) g(p)^{k-1}+b_{2}(z(p)) g(p)^{k-2}+\cdots+b_{k}(z(p)) .
$$

We then define $f_{1}$ in $H^{\infty}\left(W_{1}\right)$ by

$$
f_{1}(p)=b_{1}\left(z_{1}(p)\right) g_{1}(p)^{k-1}+b_{2}\left(z_{1}(p)\right) g_{1}(p)^{k-2}+\cdots+b_{k}\left(z_{1}(p)\right) .
$$

Then $f=f_{1} \cdot \Phi$.

In general the existence of nonconstant bounded analytic functions is not a property of the ideal boundary of an open Riemann surface. (See, e.g., Sario and Nakai [11, p. 92].) However, Widom's condition is a property of the ideal boundary. Let $W$ be a Myrberg surface, 
and let $W^{\prime}$ be an open Riemann surface obtained obtained from $W$ by changing the conformal structure on a compact subregion. If $m(W)>0$, then $m\left(W^{\prime}\right)>0$ and so $H^{\infty}\left(W^{\prime}\right)$ separates the points of $W^{\prime}$. On the other hand if $m(W)=0$, then $m\left(W^{\prime}\right)=0$ and $W^{\prime}$ may carry no nonconstant bounded analytic functions.

We can construct a class of examples of Myrberg surfaces as follows: Let $\left\langle x_{n}\right\rangle$ be a strictly increasing sequence of positive real numbers converging to 1 . Let $W_{1}$ and $W_{2}$ be two copies of the open unit disc each slit along the segments $\left[x_{2 n-1}, x_{2 n}\right], n=1,2, \ldots$. Let $W$ be the Riemann surface which results from joining $W_{1}$ and $W_{2}$ crosswise along these segments. There is a natural projection $z$ from $W$ onto the open unit disc $U$ which makes $W$ into a Myrberg surface over $U$ with a branch point of order two over each of the points $x_{n}, n=1,2, \cdots$. According to Theorem $1, H^{\infty}(W)$ separates the points of $W$ if and only if $\Sigma\left(1-x_{n}\right)<\infty$. According to Theorem 2, if $\Sigma\left(1-x_{n}\right)=\infty$, every bounded analytic function on $W$ is lifted from a bounded analytic function on $U$. Of course this example can be discussed directly without the use of Theorems 1 and 2 .

3. The maximal ideal space of $H^{\infty}(W)$. Our main result in this section is that if $m(W)>0$ then the natural mapping of $W$ into the maximal ideal space $M$ of $H^{\infty}(W)$ is a homeomorphism of $W$ onto an open subset of $M$. To prove this we adapt an argument Stout [12], p. 159, used on plane domains. In the course of our proof we must show that $H^{\circ}(W)$ separates the points of $W$ and we must use a fact about analytic structure in maximal ideal spaces. We then use the result to study representing measures of points in $W$.

Since $H^{\infty}(W)$ separates the points of $W$, we can embed $W$ as a subset of $M$. We assign to the point $p$ of $W$ the maximal ideal of those functions vanishing there. In this way $W$ acquires two topologies: its usual topology as a Riemann surface and the Gelfand topology it inherits from $M$. The Gelfand topology is the weaker, i.e., the embedding is continuous.

In order to investigate the relationship between these topologies, we shall use a fact about analytic structure in maximal ideal spaces. Let $A$ be a commutative Banach algebra with identity and let $\mathscr{M}$ be the maximal ideal space of $A$. Let $\varphi$ be in $\mathscr{A}$. A derivation of type $(I, \varphi)$ is a bounded linear operator $T$ on $A$ such that $\varphi^{\circ} T$ is not trivial and

$$
T(f g)=\varphi(f) T g+g T f
$$

for all $f, g$ in $A$. Banachewski [1] (cf. Bishop [2]) has proved that 
if $A$ admits a derivation of type $(I, \varphi)$ then there is an open neighborhood of $\varphi$ in $\mathscr{C}$ which is homeomorphic to an open disc in the complex plane.

To see that $H^{\infty}(W)$ admits a derivation of type $(I, q)$ at each point $q$ of $W$, we fix a bounded function $\Psi$ in $H(W, \chi)$ such that $\Psi(q) \neq 0$. Define $T$ by the formula

$$
T f=[f-f(q)] \Psi \Phi^{-1}
$$

for $f$ in $H^{\infty}(W)$. Now $\Psi \Phi^{-1}$ is single-valued, has a simple pole at $q$, and is bounded outside any neighborhood of $q$. Hence $T$ is a bounded linear operator on $H^{\infty}(W)$. The derivation identity

$$
T(f g)=f(q) T g+g T f
$$

for $f, g$ in $H^{\infty}(W)$ is easily verified.

It now follows immediately from Banachewski's theorem that there is an open neighborhood $V$ of $q$ in the Gelfand topology which is homeomorphic to an open disc in the plane. Now $V \cap W$ is open in the usual topology on $W$, hence there is an open parameter disc $D$ around $q$ with $D \subset(V \cap W)$. By invariance of domain, $D$ is open in the Gelfand topology on $W$. Therefore the usual topology and the Gelfand topology coincide, and $W$ is open in the Gelfand topology. We state this result as a theorem.

THeORem 3. Let $W$ be an open Riemann surface with $m(W)>0$. Let $M$ be the maximal ideal space of $H^{\infty}(W)$. Then $W$ is (homeomorphically embedded as) an open subset of $M$.

Theorem 3 allows us to extend the methods used to treat linear extremal problems in plane domains to open Riemann surfaces satisfying Widom's condition. The development can be carried out as in Gamelin [6] and yields the same theorems on the uniqueness and unimodularity of extremal functions. The only change necessary is that the local maximum modulus principle must be used in the proof of Theorem 2.2 of [6]. (The author thanks the referee for pointing out this.) Alternatively, Theorem 2.2 follows from Banachewski's theorem by a refinement of the argument we used in proving Theorem 3.

\section{REFERENCES}

1. B. Banachewski, Analytic discs in the maximal ideal sdace of a Banach algebra, Bull. Acad. Polonaise Sci. Ser. Sci. Math. Ast. Phy., 14 (1965), 137-144. 
2. E. Bishop, Analyticity in certain Banach algebras, Trans. Amer. Math., 102 (1962), 507-544.

3. S. Fisher, On Schwarz's Lemma and inner functions, Trans. Amer. Math. Soc., 138 (1969), 229-240.

4. The moduli of extremal functions, Michigan Math. J. 19 (1972), 179-183.

5. T. W. Gamelin, Uniform Algebras, Prentice-Hall, Englewood Cliffs, N. J., 1969, $257 \mathrm{pp}$.

6. - Extremal Problems in arbitrary domains, Michigan Math. J., 20 (1973), $3-12$.

7. L Lectures on $H^{\infty}(D)$, Notas de Mathemática, No. 21, Universidad Nacional de La Plata, 1972.

8. S. Ya. Havinson, Analytic capacity of sets, joint non-triviality of various classes of analytic functions, and the Schwarz lemma in arbitrary domains, Amer. Math. Soc., Trans, Series 2, 43 (1964), 215-266.

9. D. Hejhal, Linear extremal problems for analytic functions, Acta Math., 128 (1972), 91-122.

10. P. J. Myrberg, Über die analytische Fortsetzung von beschränkten Funktionen, Ann. Acad. Sci. Fenn., Ser AI, No. 58 (1949), 7 pp.

11. L. Sario and M. Nakai, Classification Theory of Riemann Surfaces, Springer-Verlag, New York-Berlin-Heidelberg, 1970, $446 \mathrm{pp}$.

12. E. L. Stout, The Theory of Uniform Algebras, Bogden and Quigley, Tarrytownon-Hudson, N.Y.-Belmont, Calif., 1971, 509 pp.

13. M. Voichick, Extreme points of bounded analytic functions on infinitely connected regions, Proc. Amer. Math. Soc., 17 (1966), 1366-1369.

14. H. Widom, The maximum principle for multiple-valued analytic functions, Acta Math., 126 (1971), 63-82.

15. - $H^{p}$-sections of vector bundles over Riemamm surfaces, Ann. of Math., (2) 94 (1971), 304-324.

Received February 1, 1974 and in revised form May 2, 1975. Much of the research for this paper was done during the academic year 1971-1972 when the author was a visitor at the Department of Mathematics, Brown University.

\section{WESLEYAN UNIVERSITY}

Current address: Department of Mathematics, University of California, Berkeley: California 94720. 



\title{
PACIFIC JOURNAL OF MATHEMATICS
}

\section{EDITORS}

RichaRd ARENS (Managing Editor)

University of California

Los Angeles, California 90024

\section{R. A. Beaumont}

University of Washington

Seattle, Washington 98105
J. DUGUNDJI

Department of Mathematics University of Southern California Los Angeles, California 90007

D. Gilbarg and J. Milgram

Stanford University

Stanford, California 94305

\section{ASSOCIATE EDITORS}
E. F. BECKENBACH
B. H. NeUmanN
F. WOLF
K. YoshIDA

\section{SUPPORTING INSTITUTIONS}

\author{
UNIVERSITY OF BRITISH COLUMBIA \\ CALIFORNIA INSTITUTE OF TECHNOLOGY \\ UNIVERSITY OF CALIFORNIA \\ MONTANA STATE UNIVERSITY \\ UNIVERSITY OF NEVADA \\ NEW MEXICO STATE UNIVERSITY \\ OREGON STATE UNIVERSITY \\ UNIVERSITY OF OREGON \\ OSAKA UNIVERSITY
}

\author{
UNIVERSITY OF SOUTHERN CALIFORNIA \\ STANFORD UNIVERSITY \\ UNIVERSITY OF TOKYO \\ UNIVERSITY OF UTAH \\ WASHINGTON STATE UNIVERSITY \\ UNIVERSITY OF WASHINGTON \\ AMERICAN MATHEMATICAL SOCIETY
}

The Supporting Institutions listed above contribute to the cost of publication of this Journal, but they are not owners or publishers and have no responsibility for its content or policies.

Mathematical papers intended for publication in the Pacific Journal of Mathematics should be in typed form or offset-reproduced, (not dittoed), double spaced with large margins. Underline Greek letters in red, German in green, and script in blue. The first paragraph or two must be capable of being used separately as a synopsis of the entire paper. Items of the bibliography should not be cited there unless absolutely necessary, in which case they must be identified by author and Journal, rather than by item number. Manuscripts, in triplicate, may be sent to any one of the editors. Please classify according to the scheme of Math. Reviews, Index to Vol. 39. All other communications should be addressed to the managing editor, or Elaine Barth, University of California, Los Angeles, California, 90024.

The Pacific Journal of Mathematics expects the author's institution to pay page charges, and reserves the right to delay publication for nonpayment of charges in case of financial emergency.

100 reprints are provided free for each article, only if page charges have been substantially paid. Additional copies may be obtained at cost in multiples of 50 .

The Pacific Journal of Mathematics is issued monthly as of January 1966. Regular subscription rate: $\$ 72.00$ a year (6 Vols., 12 issues). Special rate: $\$ 36.00$ a year to individual members of supporting institutions.

Subscriptions, orders for back numbers, and changes of address should be sent to Pacific Journal of Mathematics, 103 Highland Boulevard, Berkeley, California, 94708.

PUBLISHED BY PACIFIC JOURNAL OF MATHEMATICS, A NON-PROFIT CORPORATION

Printed at Kokusai Bunken Insatsusha (International Academic Printing Co., Ltd.), 8-8, 3-chome, Takadanobaba, Shinjuku-ku, Tokyo 160, Japan.

Copyright (C) 1975 by Pacific Journal of Mathematics Manufactured and first issued in Japan 


\section{Pacific Journal of Mathematics}

\section{Vol. 59, No. $2 \quad$ June, 1975}

Aharon Atzmon, A moment problem for positive measures on the unit disc ........

Peter W. Bates and Grant Bernard Gustafson, Green's function inequalities for

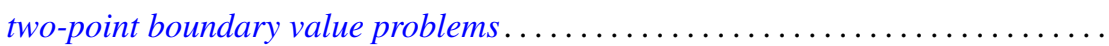

Howard Edwin Bell, Infinite subrings of infinite rings and near-rings ...........

Grahame Bennett, Victor Wayne Goodman and Charles Michael Newman, Norms of

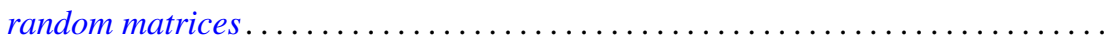

Beverly L. Brechner, Almost periodic homeomorphisms of $E^{2}$ are periodic.........

Beverly L. Brechner and R. Daniel Mauldin, Homeomorphisms of the plane ........

Jia-Arng Chao, Lusin area functions on local fields ......................

Frank Rimi DeMeyer, The Brauer group of polynomial rings ...............

M. V. Deshpande, Collectively compact sets and the ergodic theory of

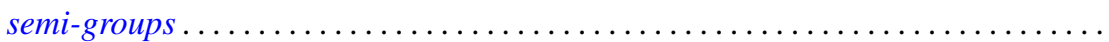

Raymond Frank Dickman and Jack Ray Porter, $\theta$-closed subsets of Hausdorff

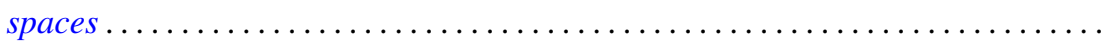

Charles P. Downey, Classification of singular integrals over a local field .......... 407

Daniel Reuven Farkas, Miscellany on Bieberbach group algebras . . . . . . . . . . . .

Peter A. Fowler, Infimum and domination principles in vector lattices . . . . . . . . . .

Barry J. Gardner, Some aspects of T-nilpotence. II: Lifting properties over

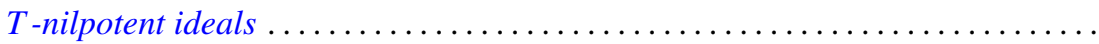

Gary Fred Gruenhage and Phillip Lee Zenor, Metrization of spaces with countable

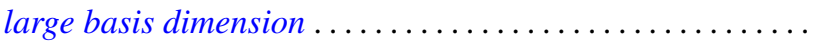

J. L. Hickman, Reducing series of ordinals...

Hugh M. Hilden, Generators for two groups related to the braid group ...

Tom (Roy Thomas Jr.) Jacob, Some matrix transformations on analytic sequence

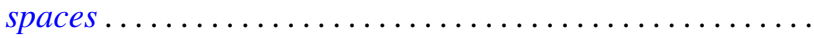

Elyahu Katz, Free products in the category of $k_{w}$-groups . .....

Tsang Hai Kuo, On conjugate Banach spaces with the Radon-Nikodým property...

Norman Eugene Liden, $K$-spaces, their antispaces and related mappings ...

Clinton M. Petty, Radon partitions in real linear spaces ........

Alan Saleski, A conditional entropy for the space of pseudo-Menger maps ....

Michael Singer, Elementary solutions of differential equations .

Eugene Spiegel and Allan Trojan, On semi-simple group algebras. I. . .

Charles Madison Stanton, Bounded analytic functions on a class of open Riemann

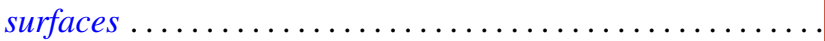

Sherman K. Stein, Transversals of Latin squares and their generalizations ....

Ivan Ernest Stux, Distribution of squarefree integers in non-linear sequences . . .

Lowell G. Sweet, On homogeneous algebras ................

Lowell G. Sweet, On doubly homogeneous algebras .......... .

Florian Vasilescu, The closed range modulus of operators ......

Arthur Anthony Yanushka, A characterization of the symplectic groups $\operatorname{PSp}(2 m, q)$

as rank 3 permutation groups... 